13024 J.B.METZLER 
Herausgegeben in Verbindung mit der Heinrich-Heine-Gesellschaft 


\title{
HEINE-JAHRBUCH 2004
}

\author{
43. Jahrgang \\ Herausgegeben von Joseph A. Kruse \\ Heinrich-Heine-Institut \\ der Landeshauptstadt Düsseldorf
}

Verlag J. B. Metzler

Stuttgart - Weimar 
Anschrift des Herausgebers: Joseph A. Kruse

Heinrich-Heine-Institut

Bilker Straße I2-I4, 40213 Düsseldorf

Redaktion: Karin Füllner und Marianne Tilch

Bibliografische Information Der Deutschen Bibliothek

Die Deutsche Bibliothek verzeichnet diese Publikation in der

Deutschen Nationalbibliografie; detaillierte bibliografische Daten sind im Internet über <http://dnb.ddb.de> abrufbar.

ISBN 978-3-476-02049-9

ISBN 978-3-476-02888-4 (eBook)

DOI 10.1007/978-3-476-02888-4

ISSN $0073^{-1692}$

Dieses Werk einschließlich aller seiner Teile ist urheberrechtlich geschützt. Jede Verwertung außerhalb der engen Grenzen des Urheberrechtsgesetzes ist ohne Zustimmung des Verlages unzulässig und strafbar. Das gilt insbesondere für Vervielfältigungen, Übersetzungen, Mikroverfilmungen und die Einspeicherung und Verarbeitung in elektronischen Systemen.

(C) 2004 Springer-Verlag GmbH Deutschland

Ursprünglich erschienen bei J. B. Metzler'sche Verlagsbuchhandung und Carl Ernst Poeschel Verlag GmbH in Stuttgart 2004

www.metzlerverlag.de

info@metzlerverlag.de 


\section{Inhalt}

Siglen $\ldots \ldots \ldots \ldots \ldots \ldots \ldots \ldots \ldots \ldots \ldots \ldots \ldots, \quad$ IX

Aufsätze

I.

"Die dritte Säule meines lyrischen Ruhmes" - Heines "Romanzero».

Beiträge des Kolloquiums im Heinrich-Heine-Institut

am I3. April 2002. Teil II . . . . . . . . . . . . . . . . . . . . I

Céline Trautmann-Waller · Bilder jüdischer Verwandlungen

in den »Hebräischen Melodien«: Metamorphose, Sublimierung

und Verklärung $\ldots \ldots \ldots \ldots \ldots \ldots \ldots \ldots \ldots \ldots \ldots \ldots \ldots \ldots \ldots \ldots$

Christian Liedtke . "... und es lachten selbst die Mumien«.

Komik und grotesker Humor in Heines »Romanzero" .......... I2

Ortwin Lämke · Küsse - Dichter - Helden - Schüsse:

Über Motivketten und Chiffren in Heines »Romanzero" ......... 3 I

Isabelle Kalinowski • „Und im Haar war ihre Kraft«. Zur Problematik

künstlerischer Heteronomie in Heines »Romanzero" ........... 49

II.

Gerhard Kaiser · Lazarus als Lyriker. Über das lyrische Werk

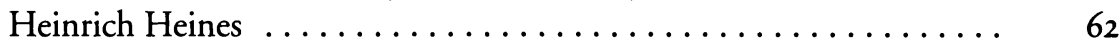

Ralph Häfner • Lyrische Maskerade. Das Problem der Gedankeneinheit

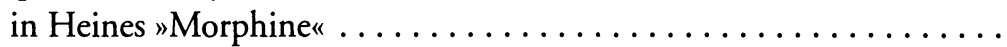


Marie-Ange Maillet . Heinrich Heine und die "Neuen allgemeinen politischen Annalen«: die Zeitschrift und ihre Bedeutung für ihren Redakteur in den Münchener Verhältnissen

Claude D. Conter · Kontinentaldiagnostik im Wandel. Heinrich Heines Positionen im Europadiskurs ................. 132

Ernest A. Menze . Herder and Heine: Reflections on Affinities . . . . . I I5O

Liu Min . Heines Lyrik in China nach 1949 . Teil $\mathrm{I} \ldots \ldots . \ldots \ldots$

III.

Gerhart Söhn • Wolfgang Menzel. Sein Leben. Teil I . . . . . . . . . . I9I

Gudrun Jäger · Friedrich Hirth - Heine-Forscher und erster Komparatist im Nachkriegsdeutschland . . . . . . . . . . . . . . .

\section{Projekte der Heinrich-Heine-Gesellschaft e.V.}

Die Heinrich-Heine-Gesellschaft in Berlin. Vorstellung in der

Landesvertretung NRW am 19. Juni $2003 \ldots \ldots \ldots \ldots \ldots \ldots \ldots$

Ingrid Bachér . Über den Umgang mit Sprache . . . . . . . . . . . . 235

Joseph A. Kruse . Ironie und Distanz bei Heine . . . . . . . . . . . $\quad 238$

Sabine Bierwirth und Irina Hundt · Bericht über die Gründung der BerlinBrandenburgischen Sektion der Heinrich-Heine-Gesellschaft e. V. . . 244

Essay-Preis der Heinrich-Heine-Gesellschaft e. V. "Heines schöner Islam" $\ldots \ldots \ldots \ldots \ldots \ldots \ldots \ldots \ldots \ldots \ldots$

Joseph A. Kruse · Begründung der Jury . . . . . . . . . . . . . . 246

Hanno Kabel . Heines schöner Islam . . . . . . . . . . . . 252

Heinrich-Heine-Institut. Sammlungen und Bestände. Aus der Arbeit des Hauses

Bernd Füllner und Christian Liedtke - Die Datenbanken des HeinrichHeine-Portals. Mit fünf unbekannten Briefen von und an Heine ... $\quad 268$ Karin Füllner • „Europäischer Heine«. Das Düsseldorfer StudierendenKolloquium 2003 mit neuen Arbeiten über Heinrich Heine . . . . . . 


\section{Buchbesprechungen}

Wolfgang Bunzel/Peter Stein/Florian Vaßen (Hrsg.) · Romantik und Vormärz. Zur Archäologie literarischer Kommunikation in der ersten Hälfte des I9. Jahrhunderts (Joseph A. Kruse) . . . . . . . . 282

Gerhard Höhn · Heine-Handbuch. Zeit, Person, Werk (Joseph A. Kruse) . . . . . . . . . . . . . . . . . . 284

Gerhard Höhn/Bernd Füllner (Hrsg.) · Deutsch-französischer Ideentransfer im Vormärz (Robert Steegers) . . . . . . . . . . . . . . . 284

Irina Hundt (Hrsg.) . Vom Salon zur Barrikade. Frauen der Heinezeit (Ariane Neuhaus-Koch) . . . . . . . . . . . . . . . . . . . . 286

Helmut Koopmann/Klaus Dieter Post (Hrsg.) · Exil. Transhistorische und transnationale Perspektiven (Joseph A. Kruse) . . . . . . . . . . .

Beate Müller (Hrsg.) · Zensur im modernen deutschen Kulturraum

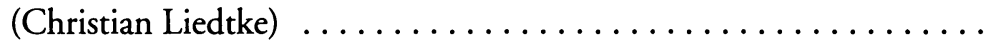

Ulrich Pongs . Heinrich Heine: Klassik, Aufklärung und Romantik (Sikander Singh) . . . . . . . . . . . . . . . . . . . . 294

Heine-Literatur 2003/2004 mit Nachträgen

Veranstaltungen des Heinrich-Heine-Instituts und der Heinrich-HeineGesellschaft e. V. Januar bis Dezember $2003 \ldots \ldots \ldots \ldots$. . . . . . . 318

Ankündigung des Düsseldorfer Studierenden-Kolloquiums 2005 . . . . . . . . 326

Kongress zum I5O. Todesjahr von Heine und Schumann im

Frühjahr 2006

Abbildungen 328

Hinweise für die Autoren

Mitarbeiter des Heine-Jahrbuchs 2004 . . . . . . . . . . . . . . . 


\section{Siglen}

I. H. Heine: Werke und Briefe

B

= Heinrich Heine: Sämtliche Schriften. Hrsg. von Klaus Briegleb. München: Hanser 1968-1976, 6 Bände (6, II = Register)

DHA

HSA

= Heinrich Heine: Historisch-kritische Gesamtausgabe der Werke. In Verbindung mit dem Heinrich-Heine-Institut hrsg. von Manfred Windfuhr. Hamburg: Hoffmann und Campe 1973-1997, I6 Bände

= Heinrich Heine: Werke, Briefwechsel, Lebenszeugnisse. Säkularausgabe. Hrsg. von den Nationalen Forschungs- und Gedenkstätten der klassischen deutschen Literatur in Weimar (seit 1991: Stiftung Weimarer Klassik) und dem Centre National de la Recherche Scientifique in Paris. Berlin und Paris: Akademie und Editions du CNRS $1970 \mathrm{ff}$.

\section{Weitere Abkürzungen}

Galley/Estermann = Eberhard Galley und Alfred Estermann (Hrsg.): Heinrich Heines Werk im Urteil seiner Zeitgenossen. Hamburg: Hoffmann und Campe 198I-1992, 6 Bände.

auf der Horst/Singh $=$ Christoph auf der Horst und Sikander Singh (Hrsg.): Heinrich Heine im Urteil seiner Zeitgenossen. Begründet von Eberhard Galley und Alfred Estermann. Stuttgart/Weimar: Metzler $2002 \mathrm{ff}$.

HJb = Heine-Jahrbuch. Hrsg. vom Heinrich-Heine-Institut Düsseldorf. Hamburg: Hoffmann und Campe 1962-1994; Stuttgart: Metzler 1995 ff.

Mende $\quad$ = Fritz Mende: Heinrich Heine. Chronik seines Lebens und Werkes. Berlin: Akademie ${ }^{1}$ 1970; ${ }^{2}$ I98I

Seifert $\quad=$ Siegfried Seifert: Heine-Bibliographie 1954-1964. Berlin und Weimar: Aufbau 1968

Seifert/Volgina = Siegfried Seifert und Albina A. Volgina: Heine-Bibliographie 1965-1982. Berlin und Weimar: Aufbau 1986

Werner $\quad$ = Michael Werner (Hrsg.): Begegnungen mit Heine. Berichte der Zeitgenossen. Hamburg: Hoffmann und Campe 1973, 2 Bände

Wilamowitz = Erdmann von Wilamowitz-Moellendorff und Günther Mühlpfordt $(\dagger)$ : Heine-Bibliographie 1983-1995. Stuttgart und Weimar: Metzler 1998

Wilhelm/Galley = Gottfried Wilhelm und Eberhard Galley: Heine-Bibliographie, [bis 1953]. Weimar: Arion 1960, 2 Bände 